\title{
Four Times of Relapse of Plasmodium vivax Malaria Despite Primaquine Treatment in a Patient with Impaired Cytochrome P450 2D6 Function
}

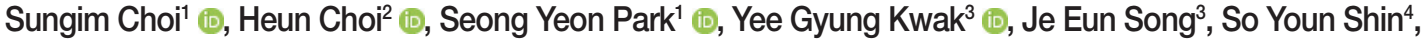 \\ Ji Hyeon Baek ${ }^{5}$, Hyun-IL Shin ${ }^{6}$, Hong Sang Oh ${ }^{\oplus}$, Yong Chan $\mathrm{Kim}^{8}$, Joon-Sup Yeom ${ }^{8}{ }^{\circ}$, Jin-Hee Han ${ }^{9}$, \\ Min Jae Kim ${ }^{10, *}$ (1)
}

\begin{abstract}
${ }^{1}$ Department of Infectious Diseases, Dongguk University Ilsan Hospital, Goyang 10326, Korea; ${ }^{2}$ Department of Infectious Diseases, National Health Insurance Service Ilsan Hospital, Goyang 10444, Korea; ${ }^{3}$ Department of Internal Medicine, Inje University Ilsan Paik Hospital, Ilsan 10380, Korea; ${ }^{4}$ Department of Infectious Diseases, International St. Mary's Hospital, College of Medicine, Catholic Kwandong University, Incheon 22711, Korea; ${ }^{5}$ Division of Infectious Diseases, Department of Internal Medicine, Inha University School of Medicine, Incheon 22332, Korea; ${ }^{6}$ Division of Vectors and Parasitic Diseases, Bureau of Infectious Disease Diagnosis Control, Korea Disease Control and Prevention Agency, Chungbuk 28159, Korea; ${ }^{7}$ Division of Infectious Disease, Department of Internal Medicine, Armed Forces Capital Hospital, Seongnam 13574, Korea; ${ }^{8}$ Department of Internal Medicine, Division of Infectious Disease, Yongin Severance Hospital, Yonsei University College of Medicine, Yongin 16995, Korea; ${ }^{9}$ Department of Medical Environmental Biology and Tropical Medicine, School of Medicine, Kangwon National University, Chuncheon 24341, Korea; ${ }^{10}$ Department of Infectious Diseases, Asan Medical Center, University of Ulsan College of Medicine, Seoul 05505, Korea
\end{abstract}

\begin{abstract}
Plasmodium vivax exhibits dormant liver-stage parasites, called hypnozoites, which can cause relapse of malaria. The only drug currently used for eliminating hypnozoites is primaquine. The antimalarial properties of primaquine are dependent on the production of oxidized metabolites by the cytochrome P450 isoenzyme 2D6 (CYP2D6). Reduced primaquine metabolism may be related to $P$. vivax relapses. We describe a case of 4 episodes of recurrence of vivax malaria in a patient with decreased CYP2D6 function. The patient was 52-year-old male with body weight of $52 \mathrm{~kg}$. He received total gastrectomy and splenectomy 7 months before the first episode and was under chemotherapy for the gastric cancer. The first episode occurred in March 2019 and each episode had intervals of 34, 41, and 97 days, respectively. At the first and second episodes, primaquine was administered as $15 \mathrm{mg}$ for 14 days. The primaquine dose was increased with $30 \mathrm{mg}$ for 14 days at the third and fourth episodes. Seven gene sequences of $P$. vivax were analyzed and revealed totally identical for all the 4 samples. The CYP2D6 genotype was analyzed and intermediate metabolizer phenotype with decreased function was identified.
\end{abstract}

Key words: P. vivax, recurrence, primaquine, CYP2D6

\section{INTRODUCTION}

Vivax malaria caused by Plasmodium vivax is the second most prevalent disease following $P$. falciparum and continues to inflict a huge burden on public health in many countries. In Korea, P. vivax is the only etiology of indigenous malaria. Annually around 500 patients have occurred mostly in northwestern area including Gyeonggi-do (Province) and the Incheon [1].

Efforts to treat and eradicate P. vivax malaria are complicated

\footnotetext{
- Received 16 December 2021, revised 8 February 2022, accepted 9 February 2022.

*Corresponding author (nahani99@gmail.com)

(c) 2022, Korean Society for Parasitology and Tropical Medicine

This is an Open Access article distributed under the terms of the Creative Commons Attribution Non-Commercial License (https://creativecommons.org/licenses/by-nc/4.0) which permits unrestricted non-commercial use, distribution, and reproduction in any medium, provided the original work is properly cited.
}

by the liver-stage parasites, called hypnozoites, that do not divide and stay dormant. If not treated properly, this hypnozoites can cause new blood-stage infections weeks to months after the initial attack and is conventionally considered to be the source of relapse in P. vivax malaria.

Currently, the only available approved drug for eliminating hypnozoites in Korea is primaquine, and the antimalarial properties of primaquine are known to be dependent on the production of oxidized metabolites by metabolization of the cytochrome P450 isoenzyme 2D6 (CYP2D6) [2]. Allelic polymorphism in the CYP2D6 gene greatly impact the enzymatic activity of CYP2D6 which is usually expressed as poor, intermediate, normal, and ultrarapid metabolizer phenotypes [3]. Patients with 'poor metabolizer (PM)' or 'intermediate metabolizer (IM)' show an impaired function in CYP2D6 enzyme, 
so even if an appropriate dose of primaquine is administered, the active metabolite is not sufficiently produced and recurrence may occur $[4,5]$.

Here, we report a case of 4 episodes of recurrence of vivax malaria and CYP2D6 analysis result.

\section{CASE DESCRIPTION}

In March 2019, a 52-year-old man living in Goyang-si, Gyeonggi-do admitted with fever, myalgia, and chill which started 3 days ago. He received total gastrectomy with splenectomy August 20th, 2018, for advanced gastric cancer. From September 2018 until 2 weeks before hospitalization, he received a total of 7 cycles of chemotherapy with capecitabine and oxaliplatin.

On admission, his axillary temperature was $38.7^{\circ} \mathrm{C}$, and his heart rate, blood pressure and respiratory rate were 120 beats per min, 100/70 mmHg and 20 breaths per min, respectively. A peripheral blood test revealed normal white blood cells (WBC) $\left(4.14 \times 10^{3} / \mathrm{mm} 3 ; 79.7 \%\right.$ neutrophil) and platelet count $\left(239,000 / \mathrm{mm}^{3}\right)$, and serum biochemistry showed elevated AST, ALT and LDH (AST, $171 \mathrm{IU} / \mathrm{L} ; \mathrm{ALT}, 67 \mathrm{IU} / \mathrm{L} ; \mathrm{LDH}$, 282 IU/L). Microscopic examination of the Giemsa-stained blood smear revealed $P$. vivax parasites $(3,146$ parasites $/ \mu l)$. His body weight was $52 \mathrm{~kg}$, and he was treated with chloroquine (total $21.7 \mathrm{mg} / \mathrm{kg}$ ) and primaquine (15 mg for 14 days). Since very low prevalence of Glucose-6-phosphate dehydrogenase (G6PD) deficiency in Koreans [7], G6PD deficiency test was not performed. Fever was cleared within 4 day and disappearance of the asexual stage parasitemia was confirmed by Giemsa-stained blood smear 5 days after chloroquine treatment. The patient was discharged on seventh hospital day and no parasite was observed in the Giemsa-stained blood smear performed 13 days after chloroquine treatment.

Thirty-four days later, the patient presented a second episode of $P$. vivax infection with similar symptoms as the first attack, but with low blood pressure $(89 / 51 \mathrm{mmHg})$. A new cycle of chemotherapy was started a week before the visit, and he was taking capecitabine. The patient had not travelled outside Goyang-si, Gyeonggi-do between these 2 attacks. Parasite density was 8,316 parasites/ $/$ l. The chloroquine was administered as total $25.8 \mathrm{mg} / \mathrm{kg}$ and primaquine was prescribed as the first episode. Parasitemia was cleared within 6 days after chloroquine treatment.

A third episode of P. vivax malaria occurred 41 days after the second episode. A new cycle of chemotherapy was started 6 days before the visit, and he was also taking capecitabine this time. Parasite density was 14,790 parasites/ $\mu$ l. Chloroquine was administered as total $20 \mathrm{mg} / \mathrm{kg}$ for the first 2 days. After then, despite a clinically significant level of chloroquine resistant malaria has not yet been identified in Korea [7], he was treated with atovaquone/proguanil 1,000/400 mg for 3 days and primaquine dose was increased as $30 \mathrm{mg}$ for 14 days. Parasitemia was cleared within 8 days after anti-malarial treatment confirmed by Giemsa-stained blood smear.

A fourth episode was diagnosed 97 days after the third epi-

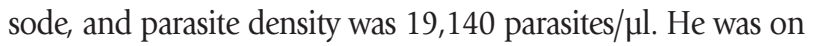
drug-holidays for gastric cancer about 2 months before the visit. He treated again with chloroquine $25 \mathrm{mg} / \mathrm{kg}$ and primaquine $30 \mathrm{mg}$ for 14 days and no parasite was observed in the Giemsa-stained blood smear performed 6 days after chloroquine treatment (Fig. 1).

Peripheral blood sample was collected at each time of diagnosis and genotyping was performed. The 4 drug resistance marker genes (pumdr1, pucrt-o, pvdhfr, and pudhps) and 3 genes with variable regions about 300 to 1,000 base pairs (pvcs, pvmsp1-F2, and pumsp1-F3) were analyzed [8]. The drug resistance genes had same alleles in all the 4 samples (Table 1). The sequences of the other 3 genes which size variable segments were totally identical among the 4 episodes (Fig. 2; Supplementary Figs. S1, S2).

CYP2D6 genotyping analysis was commissioned to SPMED Co.,Ltd., Busan, South Korea. Patient DNA was isolated and purified from blood samples using SPMED ${ }^{\mathrm{TM}}$ Genotyping Kit: CYP2D6 (in vitro license No. 20-297, SPMED Co., Ltd., Busan, Korea) according to the manufacturer's instructions and CYP2D6 *2, *3, *4, *5 (deletion), *6, *9, *10, *14, *17, *18, $* 21,{ }^{*} 29, * 41, * 49, * 52, * 60, *$ XN (duplicate) were analyzed. The patient had ${ }^{*} 10 \mathrm{~B} /{ }^{*} 10 \mathrm{~B}$ allele corresponding to the intermediate metabolizer with decreased CYP2D6 function [9].

\section{DISCUSSION}

We described a patient who experienced the 4 episodes of recurrent vivax malaria in Korea with the last relapse occurring at 171 days after the first attack. As far as we know, this is the largest number of recurrences within the same year in Korea. Our case reminds several factors regarding the treatment of malaria in Korea.

Recurrent episodes of malaria can be divided into the recru- 


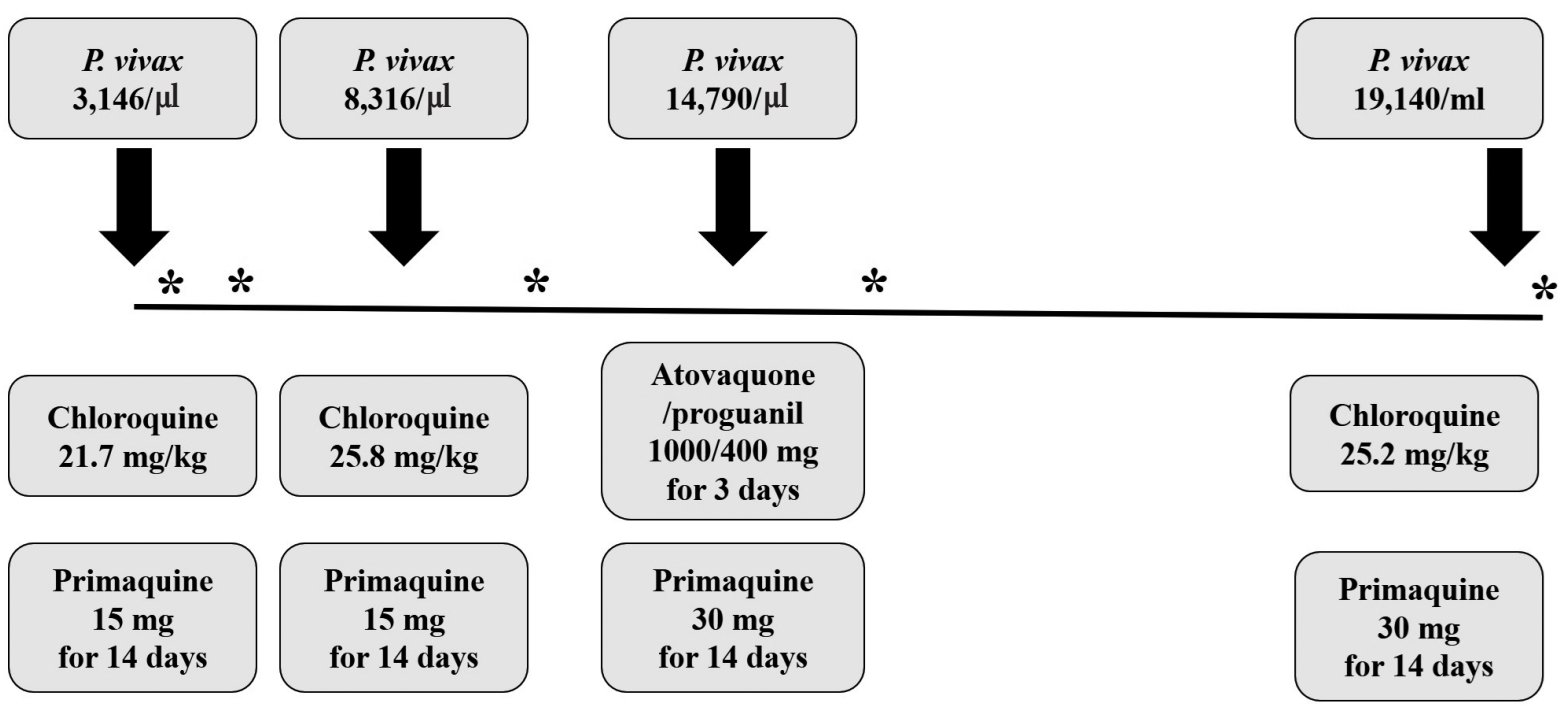

\section{* : Confirmed disappearance of parasitemia}

Fig. 1. Clinical course and parasite density, dosage of schizonticide and primaquine in the patient with recurrent vivax malaria.

A

pves

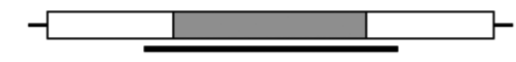

pvmsp1

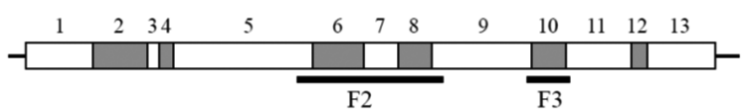

B

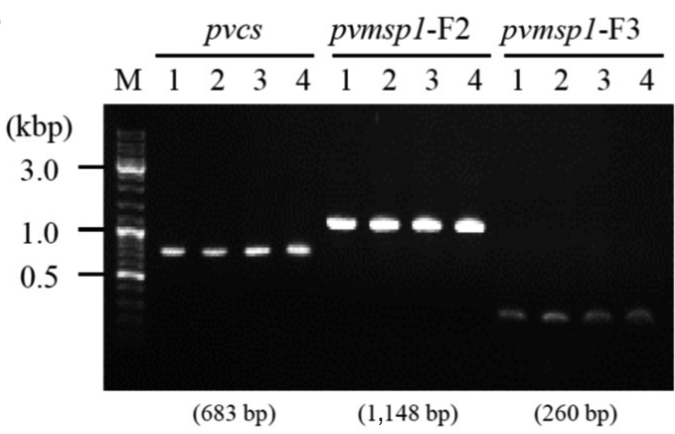

Fig. 2. Size variable genes PCR and sequencing analysis among 4 episodes. (A) Schematic representation of the pvcs and pvmsp1 gene for the localization of conserved segments (white boxes) and variable segments (grey boxes). The sequencing region is indicated by the horizontal bold lines. (B) the pvcs and pvmsp1 size variable segments PCR result. The result showed identical amplicon size among 4 episodes.

Table 1. Analysis of drug resistance genes single nucleotide polymorphisms (SNPs) from the samples of 4 recurrences

\begin{tabular}{llll}
\hline & Molecular marker & Single/multiple mutations (Allele) & No. (\%) \\
\hline Drug resistance marker & & & $4(100)$ \\
CQ & pvmdr1 & Single (F1076L) & $4(100)$ \\
& pvcrt-o & Wild type & $4(100)$ \\
Antifolate & pvdhfr & Single (S117N) & $4(100)$ \\
& pvdhps & Wild type & $4(100)$ \\
Amodiaquine+SP & pvmdr1 & Single (F1076L) & $4(100)$ \\
& pvdhfr & Single (S117N) & 4 \\
\hline
\end{tabular}

$\mathrm{CQ}$, chloroquine; SP, sulfadoxine-pyrimethamine.

descence, relapse, or re-infection. Recrudescence is the recurrent clinical malaria originating from the remnant blood stage parasites despite the anti-malaria drug treatment. In our pa- tient, the eradication of the blood stage parasites was confirmed with the microscopic exam and thus recrudescence is unlikely the cause of the recurrent episodes. There is no exact 
experimental method to distinguish the relapse from the re-infection since that relapse may originate from activation of parasite clones identical to those of the primary infections (homologous) or genetically distinct clones (heterologous) [10]. However, the sequencing data of the 7 genes, including 3 genes of variable region, from each episode revealed to be totally identical, suggesting homologous clonal relapse rather than the re-infection. This is also interesting since the studies performed in the tropical area suggest different distribution between the homologous and the heterologous hypnozoites reactivation [11].

Primaquine is currently used as hypnozoitocidal drug in Korea. The factor most strongly associated with relapse of malaria after primaquine treatment is the total amount of primaquine administered to the patient [12]. In this patient, primaquine was prescribed as $15 \mathrm{mg}$ per day $(0.29 \mathrm{mg} / \mathrm{kg} /$ day $)$ for 14 days in the first and the second episodes and as $30 \mathrm{mg}$ per day $(0.58 \mathrm{mg} / \mathrm{kg} /$ day $)$ for 14 days in the third and fourth episodes. In the third episode, even high-dose primaquine (8.1 $\mathrm{mg} / \mathrm{kg}$ total) failed to prevent the recurrence.

Anti-malarial activity of the primaquine is also known to be dependent on the production of the oxidized metabolites by the CYP2D6 isoenzyme in the liver. Thus, reduced CYP2D6 activity could have been associated with the reduced primaquine activity resulting in recurrent episodes of malaria. This patient was identified to have CYP2D6*10B/*10B allele being an IM and having $<50 \%$ activity of CYP2D6. Similar recurrent cases and studies with reduced CYP2D6 activities were also reported from the other countries $[13,14]$. Also, reviewing the patient's medical history, no CYP2D6 inhibitors such as fluoxetine [15] were administered while taking primaquine. We speculated that this genetic background could have been the major factor causing the unusual multiple episodes of vivax malaria in this patient.

This patient was under treatment for the gastric cancer. He received the total gastrectomy with splenectomy 7 months before the first episode and was under adjuvant chemotherapy during his recurrent malaria period. These host condition could have affected the long disease course. A case of severe recurrence of vivax malaria after splenectomy was reported [16], and recent a prospective study conducted in Indonesia reported that splenectomy was associated with a high risk of malaria, especially vivax malaria [17]. The association of the patient's splenectomy state with recurrence of vivax malaria cannot be completely excluded. There is no obvious clinical evidence that administration of a cytotoxic agent increases the risk of malaria relapse.

Our study has several limitations. We only present the clinical and microbiological information of only one patient. To generalize the effect of CYP2D6 on malaria recurrence, more study with larger population is needed. Second, administration of chloroquine and primaquine were not directly observed and the drug compliance could not be monitored. Third, we only examined the sequence of 7 genes of the $P$. vivax and only 3 of them contained variable region. More detailed analysis such as whole genome sequencing could have more power to differentiate more closely related $P$. vivax clones. Despite these limitations, our study clearly shows recurrence of vivax malaria is causing significant morbidity and more research about the relapse of $P$. vivax in South Korea is urgently needed.

\section{ACKNOWLEDGMENT}

The CYP2D6 genotyping was performed as part of the other study approved by ethical committees of each hospital (Asan Medical Center: 2018-0650, Dongguk University Ilsan Hospital: 2019-08-004-002).

\section{CONFLICT OF INTEREST}

The authors declare that they have no conflict of interest related to this study.

\section{REFERENCES}

1. Chai JY. History and current status of malaria in Korea. Infect Chemother 2020; 52: 441-452. https://doi.org/10.3947/ic.2020. 52.3.441

2. Potter BM, Xie LH, Vuong C, Zhang J, Zhang P, Duan D, Luong TL, Bandara Herath HM, Dhammika Nanayakkara NP, Tekwani BL, Walker LA, Nolan CK, Sciotti RJ, Zottig VE, Smith PL, Paris RM, Read LT, Li Q, Pybus BS, Sousa JC, Reichard GA, Marcsisin SR. Differential CYP 2D6 metabolism alters primaquine pharmacokinetics. Antimicrob Agents Chemother 2015; 59: 23802387. https://doi.org/10.1128/AAC.00015-15

3. Marcsisin SR, Reichard G, Pybus BS. Primaquine pharmacology in the context of CYP 2D6 pharmacogenomics: Current state of the art. Pharmacol Ther 2016; 161: 1-10. https://doi.org/10.1016/ j.pharmthera.2016.03.011

4. Bennett JW, Pybus BS, Yadava A, Tosh D, Sousa JC, McCarthy WF, Deye G, Melendez V, Ockenhouse CF. Primaquine failure and cy- 
tochrome P-450 2D6 in Plasmodium vivax malaria. N Engl J Med 2013; 369: 1381-1382. https://doi.org/10.1056/NEJMc1301936

5. Baird JK, Louisa M, Noviyanti R, Ekawati L, Elyazar I, Subekti D, Chand K, Gayatri A, Instiaty, Soebianto S, Crenna-Darusallam C, Djoko D, Hasto BD, Meriyenes D, Wesche D, Nelwan EJ, Sutanto I, Sudoyo H, Setiabudy R. Association of impaired cytochrome P450 2D6 activity genotype and phenotype with therapeutic efficacy of primaquine treatment for latent Plasmodium vivax malaria. JAMA Netw Open 2018; 1: e181449. https://doi. org/10.1001/jamanetworkopen.2018.1449

6. Goo YK, Ji SY, Shin HI, Moon JH, Cho SH, Lee WJ, Kim JY. First evaluation of glucose-6-phosphate dehydrogenase (G6PD) deficiency in vivax malaria endemic regions in the Republic of Korea. PLoS One 2014; 9: e97390. https://doi.org/10.1371/journal. pone.0097390

7. Im JH, Huh K, Yoon CG, Woo H, Lee JS, Chung MH, Klein TA, Jung J. Malaria control and chemoprophylaxis policy in the Republic of Korea Armed Forces for the previous 20 years (19972016). Malar J 2018; 17: 1-9. https://doi.org/10.1186/s12936018-2449-4

8. Imwong M, Pukrittayakamee S, Grüner AC, Rénia L, Letourneur F, Looareesuwan S, White NJ, Snounou G. Practical PCR genotyping protocols for Plasmodium vivax using Pvcs and Pvmsp1. Malar J 2005; 4: 20. https://doi.org/10.1186/1475-2875-4-20

9. Caudle KE, Sangkuhl K, Whirl-Carrillo M, Swen JJ, Haidar CE, Klein TE, Gammal RS, Relling MV, Scott SA, Hertz DL, Guchelaar HJ, Gaedigk A. Standardizing CYP2D6 genotype to phenotype translation: consensus recommendations from the clinical pharmacogenetics implementation consortium and dutch pharmacogenetics working group. Clin Transl Sci 2020; 13: 116-124. https://doi.org/10.1111/cts.12692

10. de Araujo FCF, De Rezende AnM, Fontes CJF, Carvalho LH, de Brito CFAJPo. Multiple-clone activation of hypnozoites is the leading cause of relapse in Plasmodium vivax infection. PLoS One 2012; 7: e49871. https://doi.org/10.1371/journal.pone.0049871

11. Imwong M, Snounou G, Pukrittayakamee S, Tanomsing N, Kim JR, Nandy A, Guthmann JP, Nosten F, Carlton J, Looareesuwan S,
Nair S, Sudimack D, Day NP, Anderson TJ, White NJ. Relapses of Plasmodium vivax infection usually result from activation of heterologous hypnozoites. J Infect Dis 2007; 195: 927-933. https:// doi.org/10.1086/512241

12. Schmidt LH, Fradkin R, Vaughan D, Rasco J. Radical cure of infections with Plasmodium cynomolgi: a function of total 8-aminoquinoline dose. Am J Trop Med Hyg 1977; 26: 1116-1128. https://doi. org/10.4269/ajtmh.1977.26.1116

13. Mat Salleh NH, Rahman MFA, Samsusah S, De Silva JR, Ng DC, Ghozali AH, Tan JH, Lai MY, Amir A, Liew JWK, Lau YL. Case report: recurrence of Plasmodium vivax malaria due to defective cytochrome P450 2D6 function in Pos Lenjang, Pahang, Malaysia. Trans R Soc Trop Med Hyg 2020; 114: 700-703. https://doi. org/10.1093/trstmh/traa042

14. Martin Ramírez A, Lombardia González C, Soler Maniega T, Gutierrez Liarte Á, Domingo García D, Lanza Suárez M, Bernal Fernández MJ, Rubio JM. Several Plasmodium vivax relapses after correct primaquine treatment in a patient with impaired cytochrome P450 2D6 function. Malar J 2020; 19: 259. https://doi. org/10.1186/s12936-020-03326-1

15. US Food and Drug Administration. Clinical drug interaction studies-Cytochrome P450 enzyme-and transporter-mediated drug interactions guidance for industry, January 2020 [Internet]; [cited 2020 June 5]. Available from: https://www.fda.gov/regulatoryinformation/search-fda-guidance-documents/clinical-druginteraction-studies-cytochrome-p450-enzyme-and-transportermediated-drug-interactions

16. Tagariello G, Sartori R, Inojosa WO, Candiotto L, Radossi P, Scarpa E, Tassinari C. Dramatic post-splenectomy onset of malaria caused by latent Plasmodium vivax in a female immigrant with severe immunological anaemia. Blood Transfus 2014; 12 : 428-430. https://doi.org/10.2450/2014.0242-13

17. Kho S, Andries B, Poespoprodjo JR, Commons RJ, Shanti PAI, Kenangalem E, Douglas NM, Simpson JA, Sugiarto P, Anstey NM, Price RN. High risk of Plasmodium vivax malaria following splenectomy in Papua, Indonesia. Clin Infect Dis 2019; 68: 5160. https://doi.org/ 10.1093/cid/ciy403 
